\title{
Mini-Open Reconstruction of Rotator Cuff of The Shoulder
}

\author{
Ibrahim Hussein, Emad Mohamed Zayed, Mohamed Ahmed Mahmoud Abd-Allah*
}

Orthopedic Surgery Department, Faculty of Medicine, Al-Azhar University

*Corresponding author: Mohamed Ahmed Mahmoud Abd-Allah, E-Mail: Barakat_2208@outlook.com, Mobile:01001936562

\begin{abstract}
Background: shoulder pain is one of the most common musculoskeletal (MSK) disorder affecting a large portion of the population. It is estimated that one in three persons will have at least only one episode of shoulder pain within their lifetime. Rotator cuff tears are very common in the general population. Radiographic studies have shown the prevalence of rotator cuff tears to be $4-13 \%$ in the population aged between $40-60$ years and $20 \%$ in population whom age between $60-70$ years and $30-50 \%$ in population above 70 years. Rotator cuff tears are a common pathology associated with degenerative changes in the shoulder joint. They cause significant disability, pain, dysfunction and poor health status and their prevalence is currently increasing within an aging population.

Aim of the Work: the aim of this work was to study the results of mini open rotator cuff repair of the shoulder joint. Patients and Methods: this study was conducted on ten patients presented to Al-Azhar University Hospital with rotator cuff tear of the shoulder. They are treated with mini-open repair technique. All our patients completed a period of at least one year of follow up. Results: a clinical success rate was found in ten patients treated by mini open technique, $70 \%$ had excellent and good results according to Constant and Murly score after one year of follow up. Conclusion: repair of rotator cuff tears by mini-open technique is one of the good viable options providing improvement in pain and function of the shoulder joint. Also it is more economic than arthroscopic repair and it does not need high learning curve as arthroscopic repair.
\end{abstract}

Keywords: Rotator Cuff, Mini-Open.

\section{INTRODUCTION}

Shoulder pain is one of the most common musculoskeletal disorders affecting a large portion of the population ${ }^{(1)}$.It is estimated that one in three persons will have at least only one episode of shoulder pain within their lifetime ${ }^{(2)}$.

Rotator cuff tears are very common in the general population. Radiographic studies have shown that the prevalence of rotator cuff tears to be $4-13 \%$ in the population aged between $40-60$ years and $20 \%$ in population whom age between $60-70$ years and 30 $50 \%$ in population above 70 years ${ }^{(3)}$.

Rotator cuff tears are one of the most common pathologies that is associated with degenerative changes in the shoulder joint. They cause significant disability, pain, dysfunction and poor health status and their prevalence is now increasing within an aging population $^{(4)}$.

The risk of rotator cuff tear is much more higher on the dominant side. Established factors which affect the outcome following rotator cuff repair include the tear size, age and time from the tear to surgery ${ }^{(5)}$.

The rotator cuff muscles are a group of four muscles including subscapularis in front, supraspinatus above and infraspinatus and teres minor from behind that fuse together to form a tendon which encompasses the head of the humerus. ${ }^{(6)}$

The rotator cuff muscles are part of the intrinsic muscles in the shoulder joint. Their function is to control the glenohumeral joint to provide the stability by dynamically positioning the scapula to place the glenoid properly opposite the humeral head as the shoulder moves. They stiffen the capsule and decrease glenohumeral translation. These functions make them susceptible to injury and tearing with time and age ${ }^{(7)}$.

Etiology of rotator cuff tears is multifactorial. Acromial morphology is implicated as contributing factor ; four types of acromion morphology are present: flat, curved, hooked and convex. An increase in rotator cuff tears was noted more with the hooked acromion type ${ }^{(8)}$

Although impingement may play a role, the pathologic effects of overuse appears to play more significant role in the development of rotator cuff tear. Overuse can cause imbalance between tendon degeneration and tendon regeneration leading to increased apoptosis of tenocytes and conversion of the tendons to the biomechanically inferior fibrocartilage. This can lead to attritional tears of the rotator cuff and mechanical failure. Also the aging process appears to increase the pathologic effects of overuse ${ }^{(9)}$.

Most patients with rotator cuff tears have gradual onset of progressive pain and weakness, with associated loss of active motion. Pain is usually present at night and is usually referred to the insertion of deltoid muscle so patient may be not able to sleep on the affected side. Other complaints include instability and weak abduction and external rotation $^{(\mathbf{1 0})}$.

The diagnosis of rotator cuff tears depends on impingement tests and the cuff muscles tests, namely Jobe test, lift-off test and external rotation stress test. Then the patient is initially evaluated radiographically by plain X-ray which may show the type of acromion, calcific deposits, glenohumeral arthritis, 
The diagnosis depends mainly on MRI study of the shoulder joint, which demonstrates the cuff lesion, fatty infiltration of the muscles and bony structural abnormalities of the shoulder joint ${ }^{(11)}$.

Surgical repair is being indicated in acute tears from the begining and in chronic tears that failed to respond to conservative measures ${ }^{(12)}$

Repair techniques have evolved from the open procedures to an arthroscopically assisted (mini-open) technique to the all arthroscopic techniques ${ }^{(13)}$.

Several studies have found good or excellent results following rotator cuff repair surgeries. It is a costly procedure and an increased role for nonoperative measures has been proposed ${ }^{(\mathbf{1 4})}$.

Surgical repair may be associated with some complications such as joint stiffness, injury to deltoid muscle or its nerve supply (axillary nerve) and recurrence of tear ${ }^{(\mathbf{1 5})}$.

\section{AIM OF THE WORK}

The aim of this work is to study the results of mini open rotator cuff repair of the shoulder joint.

\section{PATIENTS AND METHODS}

Between November 2016 and October 2017,ten patients with rotator cuff tear presented to Al-Azhar University hospitals (El-Hussein and Sayed Galal hospitals) were included in a prospective manner.The study was started after ethical committee approval .An informed consent was signed after full explanation of the procedure for each patient. All our patients completed a period of at least one year of follow up.

\section{The inclusion criteria include:}

1- Patient suffering from symptomatic reparable complete rotator cuff tears.

2- Their MRI examination shows small to medium size cuff tear with no or little fatty infiltration of cuff muscles.

\section{The exclusion criteria include:}

1- Patients with partial rotator cuff tears.

2- Patients with large or irreparable massive tears.

3- Patients with moderate or marked fatty infiltration of cuff muscles (stage 3 or 4).

\section{Clinical assessment:}

- Neer impingement test, Hawkins Kennedy test.

- Tests of cuff muscles: Jobe test, lift-off test, external rotation stress.

\section{Mini-open repair technique}

After finishing diagnostic shoulder arthroscopy, we perform the decompression and dealing with associated joint pathologies if present. With arm suspended in $45^{\circ}$ abduction and slight forward flexion. The glenohumeral joint inspected from the posterior portal and the anterior one is used for the tools. Intraarticular surgery may include synovectomy, trimming of labral tears, and debridment of loose fragments of rotator cuff tears.

Then we examine biceps tendon. Its lesions, if present, are in the intertubercular groove. If biceps tendon has a structural abnormality (such as tear, atrophy, or enlargement), either tenotomy or tenodesis is performed.

Subacromial bursectomy is then performed from the same portals. A blunt trocar is then directed through the posterior skin incision toward the posterolateral edge of acromion.Then the under surface of posterolateral aspect of acromion is palpated with the trocar. Trocar is then advanced to anterolateral aspect of subacromial space. Firstly , visualization is usually difficult if there is bursitis or fibrosis. This must be dealt with until a lateral portal is done for bursectomy.

Instruments are then introduced through the lateral skin portal. An electrocautery is used to maintain the heamostasis, and to remove all soft tissues from the acromial undersurface, with aid of soft tissue shaver. This is to remove all the tissues of the subacromial bursa and clearing field for later rotator cuff repair.

Decompression is then carried out with a 5.5 acromionizer burr. With resection of the anterior 0.7-1.0 $\mathrm{cm}$ of the acromion to the coronal level of the anterior aspect of the distal clavicle, and to thinning the acromion for an additional $2.0 \mathrm{~cm}$ in the posterior aspect. After the acromioplasty, coracoacromial ligament is then transected with electrocautery. On completion of the subacromial decompression, the arthroscope remains in the posterior portal and an electrocautery is then introduced through the lateral portal to remove all soft tissues and fat from around the undersurface of the acromioclavicular (AC) joint including The AC joint with its inferior capsule.

It is identified with an 18-gauge spinal needle passed from above the joint. The soft tissue shaver is used to remove all the tissues from the medial border of the acromion and the AC joint. A 5.5 burr is inserted from the lateral portal to start resection of distal end of the clavicle. The under surface of the distal end of the clavicle is burred level with the subacromial decompression. At this point, the burr may be inserted through the anterior portal.

Then, after finishing the decompression, the tear is visualized, and its location and size determined. Also, subscapularis is carefully visualized to determine its integrity.

Rotator cuff mobilization begins with an intraarticular release. This is done with the scope in the posterior portal and the electrocautery in the anterior portal. The release is performed by using the electrocautery to release adhesions between the 
undersurface of the rotator cuff and the superior portion of glenoid labrum. The release begins from anterior to posterior. A release around the the posterior, anterosuperior, and posterosuperior aspects of the labrum is then done all the distance to the posterior portal.

At this point, shaver is placed through the lateral portal and debridement of the greater tuberosity is done to prepare for the lateral cuff repair. The greater tuberosity is decorticated gently and soft tissue is debrided. A formal bone trough is not created. Then , the arthroscopic shaver is used to remove any of the torn cuff edges that appears to be nonviable.

Next, a grasper is inserted through the lateral portal and used to pull tear edges to the repair location and to assess the mobility of the cuff tendon.

In some patients, additional release is found to be important. If so, the scope is withdrawn and introduced from the lateral portal. An electrocautery is placed through the anterior portal and placed down to the base of the coracoid. The coracohumeral ligament is then released down to the base of coracoid. A sharp debridement is done from the base of coracoid laterally to the bicipital groove as a rotator cuff interval release.

At this point, mini-open approach is started. The lateral portal incision is extended to a length of 3 or $4 \mathrm{~cm}$. Then subcutaneous tissue is undermined to expose the underlying fascia. Deltoid muscle is then split in line with its fibers, incorporating the arthroscopic puncture site. This split is performed up to the acromion and down for about 3 to $4 \mathrm{~cm}$. rotation of arm permits different portions of the cuff to be visualized.

Bone to tendon fixation is now done. Simple stitches through suture anchors, are done.

Closure in layers of the deltoid fascia, subcutaneous tissue then skin is done. The other arthroscopic portals are also sutured and dressing of all incisions is done.

The patient is put in an arm sling with abduction pillow.

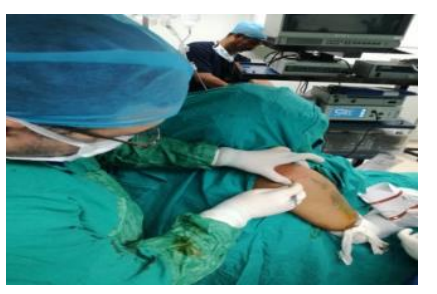

a

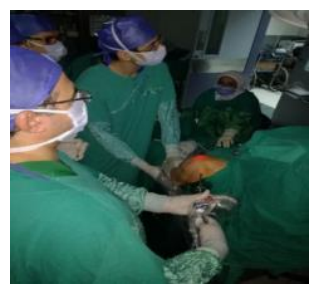

b

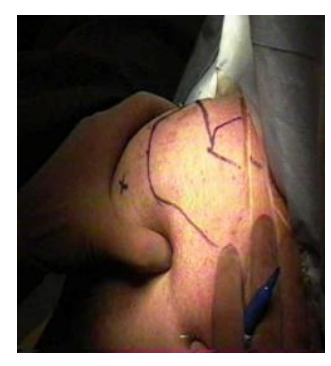

C

Figure (1): a-c: Preparation and portal placement

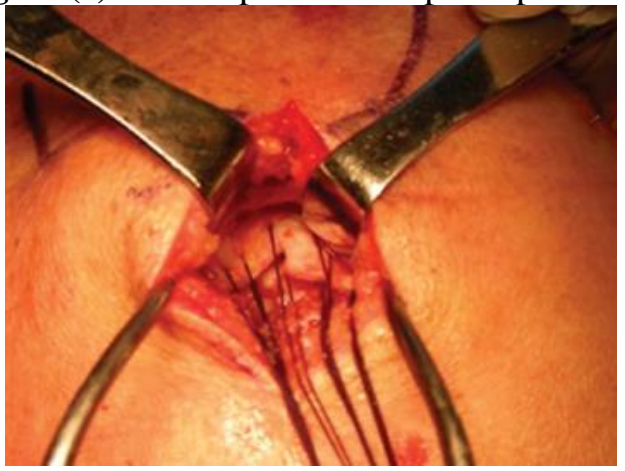

Figure (2): The cuff tear is exposed, and fixed with suture anchors.

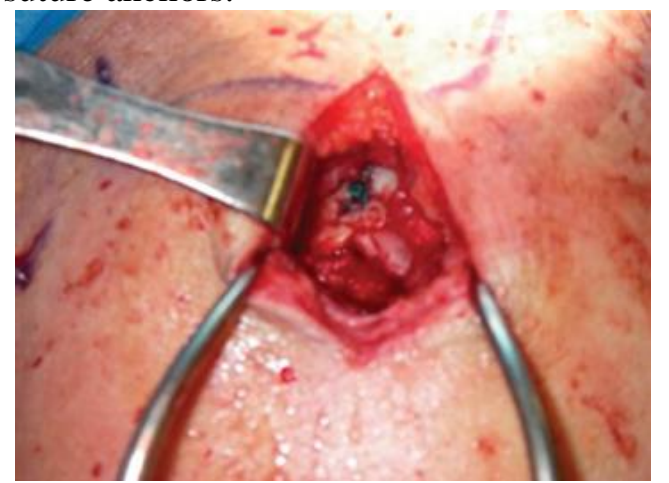

Figure (3): After repair, the deltoid can be reapproximated.

The documented preoperative, operative and postoperative follow up data for all patients were collected and reviewed and the outcome of surgery was evaluated.

The study was approved by the Ethics Board of Al-Azhar University.

\section{Statistical Method}

Data was presented as mean and standard deviation (+/- SD) for quantitative parametric data, and median and interquartile range for qualitative non parametric data. Frequency and percentage was used for presenting qualitative data. Suitable analysis was done according to the type of data obtained. Student $\mathrm{T}$ test or Mann Whitney test was used to analyze quantitative data while chi square test and fisher exact test was used to analyze qualitative data. P- value: level of significance. 
RESULTS

Mini-Open Reconstruction of Rotator Cuff of The Shoulder

Table (1): Constant- Murley score sheet of our ten patients pre and postoperative

\begin{tabular}{||c|c|c|}
\hline & Preoperative & Postoperative \\
\hline $\mathbf{1}$ & 52.0 & 77.0 \\
\hline $\mathbf{2}$ & 51.0 & 74.0 \\
\hline $\mathbf{3}$ & 54.0 & 92.0 \\
\hline $\mathbf{4}$ & 53.0 & 79.0 \\
\hline $\mathbf{5}$ & 53.0 & 68.0 \\
\hline $\mathbf{6}$ & 54.0 & 80.0 \\
\hline $\mathbf{7}$ & 51.0 & 76.0 \\
\hline $\mathbf{8}$ & 56.0 & 82.0 \\
\hline $\mathbf{9}$ & 55.0 & 80.0 \\
\hline $\mathbf{1 0}$ & 53.0 & 59.0 \\
\hline Final score & 53.2 & 76.7 \\
\hline
\end{tabular}

At the end of follow up ,final total score was recorded according to Constant and Murley score, improvement of all our patients was noticed and all patients were satisfied.

Preoperative, 10 patients had poor results and postoperative final score was excellent in one patient $10 \%$ of cases(91-100) and was good in 6 patients $60 \%$ of cases(76-90)and was fair in two patients $20 \%$ of cases(61-75) and was poor in only one patient $10 \%$ of cases(up to 60).

Final score preoperative was 53.2 and improved to 76.7 postoperative.

Table (2): Detailed postoperative score sheet of the ten patients according to Constant and Murley score:

\begin{tabular}{|c|c|c|c|c|c||}
\hline Patients & Pain & Activity of daily living & Range of motion & Power & Final score \\
\hline 1 & 10.0 & 14.0 & 32.0 & 21.0 & 77 \\
\hline 2 & 10.0 & 12.0 & 30.0 & 22.0 & 74 \\
\hline 3 & 15.0 & 18.0 & 36.0 & 23.0 & 92 \\
\hline 4 & 10.0 & 16.0 & 30.0 & 23.0 & 79 \\
\hline 5 & 5.0 & 14.0 & 26.0 & 23.0 & 68 \\
\hline 6 & 10.0 & 16.0 & 32.0 & 22.0 & 80 \\
\hline 7 & 10.0 & 12.0 & 30.0 & 24.0 & 76 \\
\hline 8 & 15.0 & 14.0 & 30.0 & 23.0 & 82 \\
\hline 9 & 10.0 & 16.0 & 32.0 & 22.0 & 80 \\
\hline 10 & 10.0 & 10.0 & 20.0 & 19.0 & 59 \\
\hline Average & 11.0 & 14.2 & 29.8 & 22.2 & 76.7 \\
\hline
\end{tabular}

Improvement in all aspects of Constant and Murley score postoperatively

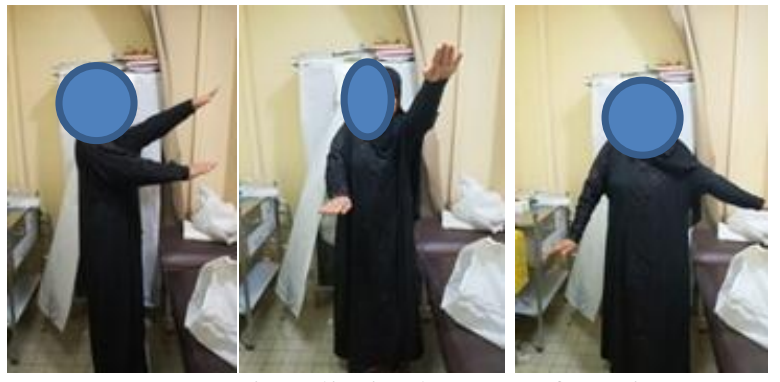

A. Preoperative: limited range of motion

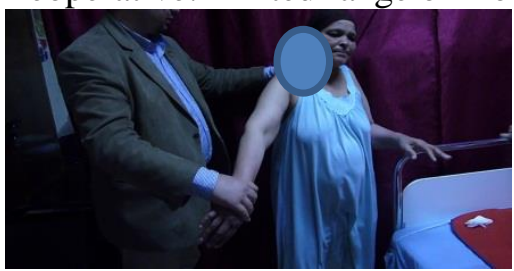

B. Positive jobe test

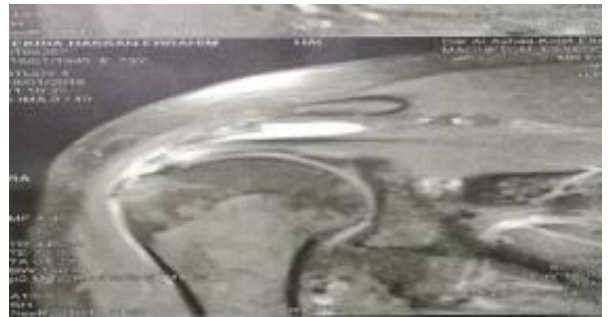

C. Preoperative MRI shoulder showing full thickness tear with no retraction.

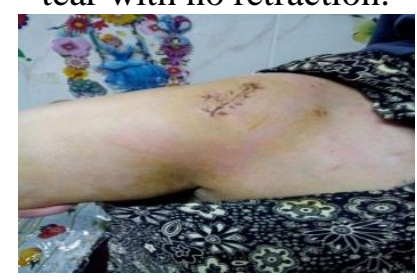

D. Mini open incision post operative 

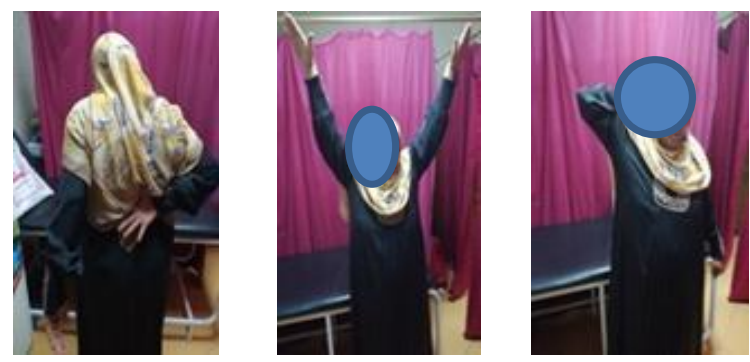

E. End of follow up: improved range of motion

\section{DISCUSSION}

Rotator cuff tears are common in general population. Pain, dysfunction, weakness and limited range of motion of the shoulder joint are the usual symptoms $^{(16)}$ The goal of any surgical repair is to decrease pain and improve function and life quality (17). This study used the mini-open repair in the treatment of cuff tears. Success rate in 10 patients treated by mini open technique was (70\%) had excellent and good results according to Constant and Murly ${ }^{(18)}$ score after one year of follow up. Which is similar to the results reported by Levy et al, ${ }^{(19)}$. The cuff surgery appears to be more effective in pain relief than in improving function of the shoulder. The pain Constant score in our study was significantly improved from a mean of 6 points preoperative to a mean of 11 points postoperative on a scale from 0 to 15. Levy et al. ${ }^{(19)}$ reported also improvement of pain score, which is similar to results published by Liu ${ }^{(20)}$, Fealy et al. (21). All of them showed marked improvement of pain. Daily activities were improved in our study from a mean of 9.6 points to a mean of 14.2 point at the end of follow up period on a scale from 0 to 20. Liu ${ }^{(20)}$ showed good or excellent function score in 84 percent of patients .Range of motion was improved in our study from a mean of 20.9 points preoperatively to a mean of 29.8 points. The strength of cuff muscles was improved in all cases from a mean of 14.5 to a mean of 22.2 on a scale from 0 to 25 . Liu ${ }^{(20)}$ showed that all patients improved by at least one grade on manual strength testing. Fealy et al . ${ }^{(21)}$ showed similar improvement.

\section{CONCLUSION}

Repair of rotator cuff tears by mini-open surgical technique is a good viable option providing improvement in pain and shoulder function. Also it is more economic than arthroscopic repair and it does not need high learning curve as arthroscopic repair.

\section{REFERENCES}

1. Struyf F, Geraets J, Noten $S$ et al. (2016): A multivariable prediction model for the chronification of non-traumatic shoulder pain: a systematic review. Pain Physician, 19:1-10.

2. Peek AL, Miller C, Heneghan NR (2015): Thoracic manual therapy in the management of non-specific shoulder pain: a systematic review. J Man Manip Ther.. 23:176-87.
3. Lehman C ,Kummer FJ, Zuckerman J(1995): The incidence of rotator cuff tears in a large population. Bull HospJt Dis ..54:30-31.

4. Tashjian R (2012): Epidemiology, natural history, and indications for treatment of rotator cuff tears. Clin Sports Med. doi; 53-57.

5. Sayampanathan A, Andrew T(2017): Systematic review on risk factors of rotator cuff tears. J Orthop Surg., 25(1).

6. DePalma AF (2008): Surgical anatomy of the rotator cuff and the natural history of degenerative periarthritis. ClinOrthopRelat Res..466:543-51.

7. Norwood LA, Barrack R, Jacobson KE(1989): Clinical presentation and radiology of complete tears of the rotator cuff. The Journal of Bone and Joint Surgery, 71(4): 499-505.

8. Budoff J, Lin C, Hong C (2016): The effect of coracoacromial ligament excision and acromioplasty on the amount of rotator cuff force production necessary to restore intact glenohumeral biomechanics. J Shoulder Elbow Surg.,25:967-972.

9. Tan M, Lam PH, Le BT et al. (2016): Trauma versus no trauma: an analysis of the effect of tear mechanism on tendon healing. J Shoulder Elbow Surg.,25(1):12-21.

10.Bhargav D, Murrell G (2004): clinical presentation and diagnosis. Australian Family Physician, 33(3):143-7.

11. Norwood LA, Barrack R, Jacobson KE(1989): Clinical presentation and radiology of complete tears of the rotator cuff. The Journal of Bone and Joint Surgery,71(4): 499-505.

12. Lambers-Heerspink FO, Hoogeslag RA, Diercks RL et al.(2011): Clinical and radiological outcome of conservative vs. surgical treatment of atraumatic degenerative rotator cuff rupture: design of a randomized controlled trial. BMC MusculoskeletDisord, 12:25.

13. Galatz LM(2000): Arthroscopic versus mini-open approach in the surgical treatment of rotator cuff tears. Current Opinion in Orthopedics, 11: 282-7.

14. Kukkonen J, Joukainen A, Lehtinen J et al.(2015): Treatment of nontraumatic rotator cuff tears: A randomized controlled trial with two years of clinical and imaging followup. J Bone Joint Surg Am.,97(21):1729-37.

15. Chelbi CM, Riley C, Murthi A(2011):Rotator cuff debate:open versus mini open versus arthroscopic. Current opinion in orthopedics ,16:263-6.

16. Ok HS, Kim BG, Choi WC(2017): Clinical Relevance of Classifying Rotator Cuff Tears Am J Sports Med., 45(1):157166.

17. Ghodadra NS, Provencher MT, Verma NN et al.(2009): Open, mini-open, and all-arthroscopic rotator cuff repair surgery: indications and implications for rehabilitation. J Orthop Sports Phys Ther .,39(2):81-9.

18. Yao M, Yang L, Cao ZY, Cheng S, Tian S, Sun Y et al.(2017): Chinese version of the Constant-Murley questionnaire for shoulder pain and disability. Health Qual Life Outcomes ,15(1): 178 .

19.Levy HJ, Uribe JW, Delaney LG(1990): Arthroscopic assisted rotator cuff repair: preliminary results. Arthroscopy ,6:55-60 .

20.Liu SH(1994): Arthroscopically assisted rotator cuff repair. J Bone Joint Surg [Br],76-B:592-5.

21. Fealy S, Kingham TP, Altchek DW(1999): Arthroscopically assisted (mini-open) rotator cuff repair. Sports Med Arthrosc Rev ., 7(2):76-84. 